\title{
What drives recommendations for practice?
}

\author{
Helen Macdonald UK research editor
}

The BMJ

Recommendations for practice can bring clarity, controversy, or confusion. Much depends on the interpretation of limited evidence, the role of commercial influence, and recognition of cultural change.

The BMJ has published two guidelines on subclinical hypothyroidism in the past 12 months whose recommendations differ. A Rapid Recommendation we published last year (doi:10. 1136/bmj.12006) was based on a systematic review and meta-analysis of 21 trials of thyroxine. Its panel strongly recommended against treating subclinical hypothyroidism in most adults, because they found high quality evidence that thyroxine made no important difference to symptoms at 12 months. They described a lack of evidence for long term benefit or harm and said that taking a pill and "lifelong" testing were likely to be burdensome.

The authors of the latest NICE guidance agree that evidence is lacking on whether thyroxine reduces cardiovascular risk in people with subclinical hypothyroidism (doi:10.1136/bmj.m41). But on the basis of their appraisal of six trials, which they deemed "generally of very low quality and equivocal," they are uncertain about whether thyroxine treatment alters quality of life. In contrast to the Rapid Recommendation panel, they make two "consider" recommendations (guideline jargon for recommendations that are uncertain). They suggest that clinicians consider giving levothyroxine to adults with a thyroid stimulating hormone (TSH) concentration of $\geq 10 \mathrm{mU} / \mathrm{L}$. They also say that clinicians should consider a six month trial in people aged over 65 years who have an elevated TSH level that is lower than $10 \mathrm{mU} / \mathrm{L}$ and who have symptoms. Without treatment, they say, TSH may not "return to the reference range," while treatment may improve symptoms and cardiovascular outcomes.

Lola Adekunle and colleagues' systematic review finds that authors with financial links to the indoor tanning industry were more likely to support indoor tanning than to be neutral or critical (doi:10.1136/bmj.m7). This may come as no surprise, but the example is unusual, say Jerod Stapleton and Joel Hillhouse in their linked editorial (doi:10.1136/bmj.m345), because the industry is focusing on promoting the benefits of its products rather than challenging concerns about its harms. This article is part of our collection on commercial interests (bmj.com/commercial-influence). If you would like to see medical research, practice, and education that is free from commercial influence, please sign our "call to action" (http:// bit.ly/2GVplHm).

Finally, a shift in medical culture drives a recommendation from Hugh Rayner and colleagues to stop writing clinical letters to other health professionals about patients' care and address them to the patients instead (doi:10.1136/bmj.m24). This is one of many changes to help dismantle medical paternalism and work towards a culture of more equal relationships with our patients. Their article includes tips on how to write such a letter. 